\title{
Introduction to the contents of issue 50:2
}

\author{
Axel Ruhe
}

Published online: 16 April 2010

(C) Springer Science + Business Media B.V. 2010

I write this in the month of March, when skiers are still rushing down the slope outside my window and we celebrated the International Women's Day on March 8. I note with appreciation that women are coming to prominence in our field of scientific endeavor. Of the 24 authors in this issue at least 8 are women, making it one of three. Last time I counted, issue 45:1, it was 6 of 25 or one of four. The share of women is expanding, let me guess that after another 5 years they will be one of two!

Let me now present the contributions to this second issue of volume 50:

Manuel Calvo, M. Pilar Laburta, J. Ignazio Montijano and Luis Rández describe a Runge Kutta method, applied to an autonomous initial value problem, which preserves a smooth Lyapunov function. This gives a new tool to compute a numerical solution that has the same regularity as the mathematical model.

Gheorghe Coman and Teodora Cătinaş develop Lagrange, Hermite and Birkhoff interpolation operators over a triangle with one curved side. Such interpolations are useful to build up finite element approximations over nonstandard regions.

Constanza Conti and Lucia Romani study how to subdivide an interval to make up spline approximations that converge to a wanted smooth function. The interest is on subdivisions with variable masks and shifted affine combinations of known symbols are used.

Sergei Gusev, Stefan Johansson, Bo Kågström, Anton Shiriaev and Andras Varga describe and compare several algorithms to solve a periodic Riccati system of linear ordinary differential equations. Two of the algorithms are of a multiple shooting character, and rely upon finding a stable invariant subspace of an associated Hamiltonian

A. Ruhe (凶)

Department of Numerical Analysis and Computer Science, NADA, Royal Institute of Technology KTH, 10044 Stockholm, Sweden

e-mail: ruhe@nada.kth.se 
system, and the third reformulates the system as a semidefinite programming problem with linear matrix constraints. Period length and number of states give clues to which of these algorithms to choose. This is illustrated by specially adapted artificial test examples and applied models from Mechanics.

Helge Holden, Nils Henrik Risebro and Hilde Sande study a front tracking model of a one dimensional immiscible flow of several isentropic gases. They show that the front tracking algorithm converges to a weak solution, provided that the initial data have a bounded total variation.

Chong-Jun Li, Vittoria Demichelis and Catterina Dagnino compute a principal value of the double integral of a function with a second order singularity in one point. A polygonal domain is subdivided into quadrilateral elements, with the singularity placed in the center of a square.

Deanna Needell studies the convergence of a randomized Kaczmarz algorithm to solve overdetermined and ill posed linear systems with noise. The interest is for tomography and image processing applications.

Stig Skelboe describes an adaptive algorithm to subdivide an implicit system of ordinary differential equations into loosely coupled subsystems. These subsystems are solved by a low order backward differentiation method. The algorithm is illustrated on a system coming from a VLSI application.

Zhe Sun, Jinping Zeng and Donghui Li solve a linear complementarity problem, where the matrix is an M matrix, with a semismooth Newton Schwarz iteration. Conditions for monotone and superlinear convergence are established.

Welcome to another issue of BIT!

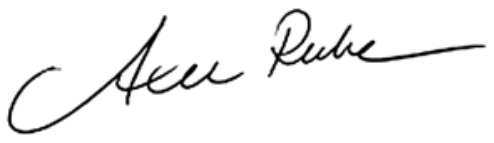

Axel Ruhe 